\title{
Sistemas productivos de comunitarios mestizos en la Costa Caribe Norte de Nicaragua
}

\author{
Jerónimo Matamoros Raudez' \\ Enrique Cordón Suárez²
}

\section{Resumen}

C sta investigación ha caracterizado los sistemas productivos, partiendo de las experiencias de las faEmilias mestizas de la comunidad de Caño Seco del Municipio de Siuna de la Costa Caribe Norte de Nicaragua. Se trata de un estudio cualitativo sustentado en un diseño etnográfico donde se suministró una encuesta, entrevista y visitas de áreas productivas a 12 familias comunitarias. Los principales resultados manifiestan: que los sistemas productivos funcionan bajo la lógica productiva de empresa familiar, en donde la mayor parte de la producción es destinada al mercado; las actividades agrícolas se centran en los cultivos anuales como: maíz, frijol, yuca y musáceas, con alta dependencia de agroquímicos, lo que incrementa los costos de producción, que a la vez provocan daños ambientales; la actividad productiva más importante es la pecuaria, enfocada en la crianza de ganado bovino con doble propósito (leche-carne). Esta ejerce gran presión sobre los recursos naturales, puesto que demanda grandes extensiones de tierra para pasturas; en la mayoría de los casos, la producción pecuaria se diversifica con la crianza de cerdos y gallinas manejados bajo un sistema extensivo poco tecnificado; $y$ otro aspecto importante, es que mujeres y niños se ven involucrados en los sistemas productivos, sin embargo, no son muy tomados en cuenta en las tomas de decisiones y tienen poco acceso a los recursos principalmente tierra.

Palabras clave: sistema productivo mestizo; valoración económica, participación de la mujer.

\section{Abstract}

This research has characterized the productive systems, starting from the experiences of the mestizo families of the community of Caño Seco of the Municipality of Siuna of Nicaragua's North Caribbean Coast. This is a qualitative study based on an ethnographic design where a survey, interview and visits of productive areas were provided to 12 community families. The main results show that the productive systems work under the productive logic of family business, where most of the production is destined to the market; Agricultural activities are focused on annual crops such as maize, beans, cassava and musaceae, with high dependence on agrochemicals, which increases production costs, which in turn causes environmental damage; The most important productive activity is livestock, focused on raising cattle with dual purpose (milk-meat). This exerts greater pressure on the natural resources, since it demands great extensions of land for pastures; in the majority of cases, the livestock production is diversified with the raising of pigs and hens handled under an extensive system little technified, and another important aspect, is that women and children are involved in productive systems, however, are not very taken into account in decision-making and have little access to resources, mainly land.

Keywords: Mestizo's productive system; Economic valuation, women's participation.

\footnotetext{
1 Ingeniero Agroforestal. Correo: jeronimoraudez@yahoo.es

2 Doctor en Agroecología Tropical. Vicerrector de la Universidad de las Regiones Autónomas de la Costa Caribe Nicaragüense-Recinto Universitario Bilwi. Correo: enrique.cordon@uraccan.edu.ni
} 


\section{Introducción}

Este estudio se enfoca en caracterizar sistemas productivos que desarrollan los comunitarios de Caño Seco de la Costa Caribe Norte de Nicaragua, así como lograr encontrar estrategias que permitan mejorar la sostenibilidad de los comunitarios. Los sistemas productivos son de gran importancia en la vida comunitaria ya que funcionan como espacio de trabajo y fuente de empleo para el sostenimiento de las familias. Por ahora los sistemas productivos se perciben como una empresa familiar.

Es importante mencionar que las actividades agrícolas de la Costa Caribe Norte son: el cultivo de granos básicos como arroz, maíz, raíces y tubérculos; y la crianza de ganado mayor y menor que son propiamente para el consumo local y nacional (FUNICA, 2009). La comunidad de Caño Seco, sitio donde se realizó esta investigación, se ubica en el municipio de Siuna, está habitada por 445 personas y agrupadas en 92 familias. La economía agropecuaria de los comunitarios de Caño Seco, estaba basada únicamente en la agricultura de subsistencia con una baja de diversidad de la producción, no obstante, en la actualidad con el incremento de la demanda de productos agrícolas y pecuarios, los sistemas productivos han cambiado a un sistema más diverso y se combinan con sistemas agrosilvopastoriles.

\section{Revisión de la literatura}

\section{Los sistemas productivos}

Un sistema de producción en un ecosistema, es el resultado de la combinación, a través del tiempo, de las fuerzas de trabajo, medios de producción, bajo la dirección de un centro de decisión Nitlapan (2010); sin embargo, HART (1980), lo describe como la interacción de varios componentes, como un conjunto de plantas, animales en un suelo y clima dado, que son manejados por el hombre con técnicas, herramientas para lograr un producto deseado.

El sistema de producción campesino es en el cual el comunitario está acostumbrado tradicionalmente a vivir, puede ser extensivo o intensivo y la característica común es que no hay criterios técnicos de manejo del ganado. El concepto de sistema de producción campesino se define a nivel de la familia y se compone de diferentes subsistemas: sistemas de cultivo, sistemas de crianza, sistemas de transformación de los productos, actividades económicas no agrícolas. Es la combinación de estos elementos constitutivos (tierra, mano de obra, capital), la que determina las estrategias productivas de la familia campesina.

La implementación o funcionamiento de cada uno de los sistemas productivos está ligado a la lógica productiva propia de la unidad familiar que persigue objetivos socioeconómicos específicos. Se considera que las decisiones relativas a la gestión del sistema son racionales, es decir que la unidad de producción moviliza medios y los utiliza de manera coherente para obtener los fines socioeconómicos y productivos deseados, esta racionalidad varía de un sistema de producción a otro según los recursos disponibles (Frédéric, 2010).

Por otro lado, los sistemas de crianza están definidos a nivel de hatos o rebaños y en ellos existen varios subsistemas. En lo interno del sistema de producción, el grado de manejo o tecnificación en éstos, va estar en dependencia de los objetivos y la visión del productor manual agropecuario.

\section{Caracterización del sistema de producción}

La caracterización de los sistemas de producción permite conocer la tecnología local de ésta, el porqué de su utilización, la posibilidad y viabilidad para implementar nuevas tecnologías (Ospina, 2008). Se debe hacer por cada uno de sus componentes por lo que no se irá a indicar solamente el tipo de tecnología que se puede generar y la forma como se puede definir, generalmente, se inicia con la información de estructura y tenencia de la tierra, la forma y clase de la producción agrícola; esta información permite tipificar o agrupar a los productores según los tipos de productos. Los objetivos de la caracterización según Ospina (2008), son:

- Conseguir información técnica de referencia sobre las prácticas productivas y la productividad en el lugar de estudio.

- Entender el proceso de toma de decisión de los productores en relación con el funcionamiento de sus sistemas de producción.

- Identificar los factores limitantes y las posibilidades de generar alternativas para los sistemas caracterizados.

En otro orden, el análisis de un sistema de producción no consiste sólo en la caracterización de cada uno de sus elementos constitutivos (aná- 
lisis de la estructura del sistema), sino también en el estudio de su funcionamiento. Con este propósito, se analizará la combinación de los diferentes elementos constitutivos al interior del sistema de producción, además, las estrategias productivas de las familias comunitarias dependen, en primera instancia, de la importancia relativa de cada uno de estos recursos. Una familia campesina que tiene una gran cantidad de tierra y poca mano de obra familiar, no seleccionará los mismos sistemas de cultivo y crianza que una que tenga mucha mano de obra disponible y poca tierra.

Más allá de la caracterización de cada elemento constitutivo del sistema de producción, es importante analizar la interdependencia de ellos. En efecto, las características de cada elemento influyen sobre los otros y por lo tanto sobre el funcionamiento global del mismo.

\section{Economía campesina}

La economía campesina está definida por la cultura y no por el mercado, los campesinos, como lo dice Lamarche (1994), no están interesados en acumular riqueza, se practica una economía que opera con una racionalidad distinta a la del capitalismo, cuya base no es la búsqueda de la ganancia, sino el mantenimiento de un equilibrio entre producción y consumo, para obtener un ingreso adecuado a las necesidades de la unidad familiar. Según Lamarche (1994), las necesidades de la unidad familiar, de acuerdo a los objetivos que se trazan, definen el modelo empresarial, el modelo de empresa familiar, el modelo de explotación campesina o de subsistencia y el modelo de explotación familiar moderna, entre las cuales la empresa familiar y la explotación campesina o de subsistencia son las más comunes.

El modelo de empresa familiar se caracteriza por la predominancia de la mano de obra familiar y porque el patrimonio y las proyecciones de producción forman parte del núcleo familiar y de sus decisiones pensadas en beneficio del mismo. El modelo de explotación campesina o de subsistencia, se caracteriza por sus técnicas tradicionales y una producción no excesiva, que también busca satisfacer solo las necesidades básicas de la familia.

\section{Relación Beneficio - Costo (B/C)}

La relación beneficio-costo es una razón que indica el retorno, en dinero, que se obtiene de cada unidad monetaria invertida. El cálculo de este indicador se realiza dividiendo el ingreso bruto entre los costos totales. Si el resultado es igual a 1 (uno), el productor o productora no pierde ni gana ya que los ingresos son iguales a los costos. Si el resultado es menor que uno, el productor o productora obtiene pérdidas y si es mayor que uno obtiene ganancias (Jaime, 1999). La fórmula para su cálculo es la siguiente:

$\mathrm{R}=\mathrm{G} / \mathrm{CU} X 100$ (Dónde: $\mathrm{R}=$ Rentabilidad; $\mathrm{G}=$ Ganancia; $C U=$ Costo unitario). En algunos casos la relación beneficio-costo se calcula utilizando el ingreso neto; en este caso, la interpretación no será la misma que en el caso anterior, debido a que al usar los ingresos netos el costo total ya ha sido deducido (Jaime, 1999).

\section{Condiciones de género en la Costa Caribe Norte de Nicaragua}

Tratar la relación de género en el país y en especial en las Costa Caribe Norte de Nicaragua, es un tema con el cual se ha vivido dentro de las familias. Sin embargo, la institucionalización del machismo con la cual se convive, no ha permitido visualizar el trabajo que realiza cada uno de los integrantes de una familia indígena y campesina (FUNICA, 2009).

Solamente un porcentaje de 15,4 de la propiedad debidamente registrada está a nombre de mujeres y 1,7 por ciento a nombre de la pareja. El 94,3 por ciento de las mujeres propietarias tiene menos de 20 manzanas de tierra versus el 33,7 por ciento de los hombres. El grupo con menor acceso a crédito en el país es el de las mujeres rurales de la Costa Caribe, quienes obtienen solamente el 18 por ciento de los préstamos otorgados y el 6,5 de la masa crediticia total (FUNICA, 2009).

\section{Métodos y materiales}

Esta investigación es de carácter cualitativa sustentada en un diseño etnográfico, porque busca recopilar información sobre el funcionamiento y lógica productiva de los sistemas de producción comunitarios. La recolección de la información se hizo mediante entrevistas, encuestas, grupos focales y visitas a las parcelas productivas. Para el desarrollo del estudio se definieron las siguientes variables: agricultura, ganadería mayor y menor, ambiente y género. 
Los participantes en esta investigación fueron 12 familias, se tratan de familias que se dedican a las actividades agrícolas y pecuarias identificados por los líderes de la comunidad. Para efectos de la investigación se seleccionaron los rubros más representativos y de mayor importancia para las familias. Subsistema agrícola: Maíz (Zea mays), Frijol (Phaseolus vulgaris), Yuca (Manihot sculenta). Subsistema pecuario: bovinos, porcinos, gallinas, equinos.

El análisis económico para los sistemas productivos se realizaron por medio de la metodología de la relación Beneficio - Costo $(B / C)$ usando la fórmula: $R=G / C U X 100$ (Dónde: $R=$ Rentabilidad; $\mathrm{G}=$ Ganancia; $\mathrm{CU}=$ Costo unitario). La valoración ambiental fue realizada a través de la observación directa dada durante las visitas de campo en las comunidades en estudio.

\section{Resultados y discusión}

En la comunidad de Caño Seco, las unidades productivas (fincas) están delimitadas y divididas por cercas (alambres), característico de un sistema de producción privado, en donde los productores dividen las áreas pecuarias y agrícolas, para evitar la introducción de ganado mayor y menor a los cultivos. Con el incremento de la producción pecuaria, la delimitación de las cercas dentro de los sistemas productivos y entre vecinos se ha aumentado.

\section{Subsistema agrícola}

Se basan principalmente en la siembra de granos básicos como: arroz (Oryza sativa), maíz (Zea mays), frijol (Paseolus vulgaris), raíces y tubérculos. Las áreas de cultivos son muy diversas en cuanto a tamaño, está en dependencia de la cantidad de miembros en la familia y la disponibilidad del recurso tierra. Son seleccionadas tomando en cuenta aspectos como topografía, que sea moderada ( $>10 \%$ de pendiente), que facilite las actividades agrícolas, que evite inundaciones o que el proceso de erosión no sea muy severo. Generalmente poseen poca disponibilidad de tierras, por tanto, destinan áreas específicas de siembra año con año. Esto imposibilita realizar prácticas de rotación de parcelas que permitan la recuperación de la fertilidad del suelo de manera natural. Este subsistema es manejado bajo un sistema semi-tecnificado, que implica el uso de herbicidas, plaguicidas y abonos químicos que de alguna manera, incrementan el rendimiento pero aumentan los costos de producción teniendo efectos negativos para el medio ambiente.

La preparación del terreno para la siembra la realizan mediante el uso de machetes, limas, y herbicidas pre emergentes como gramozone, glifosato, 2-4 D, días antes de la siembra, con el objetivo de eliminar las malezas y asegurar que el terreno esté libre de ellas al momento de la siembra. La razón de ello, según los productores, está ligada a que usan un sistema de rotación de cultivo anual, lo que conlleva a que las parcelas estén habitadas por plantas o matorrales de ciclos muy cortos. La siembra se realiza mediante el método al espeque, utilizando semillas mejoradas principalmente de ciclos cortos, mediadas de adaptación al cambio climático.

El maíz es cultivado en dos ciclos productivo (primera y postrera), el primero corresponde entre los meses de mayo-agosto y el segundo entre octubre-enero. La siembra la realizan formando surcos, esto facilita realizar con eficiencia el manejo agronómico, que incluyen actividades de control químico de malezas, cultural y la fertilización. El manejo agronómico de este rubro incluye actividades como; fertilización con N-P-K a los 15 días después de la siembra, con dosis de 1 onza por planta, seguido de una segunda aplicación con la misma fórmula a los 50 días después de la siembra. El control de maleza lo realizan empleando métodos químicos mediante el uso de herbicidas 2-4 D y gramozone con dosis de 2 onzas por cada bombada de 20 litros. Para el empacado o almacenamiento del maíz usan diferentes métodos, uno de ellos es empacar lo granos en bolsas plásticas quintaleras acompañado con un $1 / 4$ de pastilla de fosfuro de aluminio, para evitar que el maíz sea atacado por pequeños insectos, principalmente gorgojos o en barriles metálicos en buen estado.

Otro de los rubros principales cultivados es el frijol, su producción es destinada al comercio y autoconsumo, siendo la actividad agrícola más importante para generar ingresos económicos. Generalmente cada familia cultiva de 1-7 manzanas, esto tiene mucha relación con el nivel de explotación de los sistemas productivos estará en dependencia de los objetivos y los recursos económicos que posea cada familia. Se cultiva en dos ciclos productivos, en apante que comprende los meses de noviembre a febrero y postrera de septiembre a noviembre. La siembra la realizan mediante el uso de semillas criollas adaptadas a la zona. 
El manejo agronómico lo realizan usando métodos químicos, los controles de malezas lo realizan mediante el uso de agroquímicos como fusilada y Flex con dosis de 1 onza por cada bombada de 20 litros, de igual manera el control de plagas, lo realizan con insecticidas químicos (Cypermetrina) aplicando 1 onza por bombada de 20 litros. El proceso post cosecha lo inician con el despolve de las semillas para su posterior almacenamiento en recipientes metálicos (barriles), que garantizan la calidad del grano sin afectación de insectos y de esta manera tener semillas disponibles para el siguiente ciclo, es un método muy económico sin riesgo de contaminación.

De igual manera, el cultivo de yuca representa una de las principales fuentes de alimento para las familias, cultivan parcelas promedios 0.2 -0.5 manzanas. La siembra se realiza en el ciclo de primera comprendiendo los meses de mayo- junio. Utilizan estacas provenientes de plantaciones anteriores de variedades criollas adaptadas a la zona y conocida por los productores por sus características fenotípicas como: yuca negra, panameña o yuca blanca. La cosecha se completa a los 8 meses después de siembra, periodo cuando el cultivo está listo.

\section{Subsistema pecuario}

Lo integran la crianza de diferentes especies de animales domésticos: bovinos, cerdos, gallinas y equinos, siendo la crianza de bovinos la actividad más importante que les genera recursos económicos. Generalmente con los recursos obtenidos ellos mejoran sus condiciones de vida. El ganado es manejado bajo un sistema extensivo semi-tecnificado con objetivos productivos de doble propósito (leche - carne), donde el mejoramiento genético que prevalece es la monta natural. Utilizan sementales de acuerdo a la capacidad económica de cada productor y del nivel de conocimiento sobre este tema.

La alimentación de esta especie se da mediante el pastoreo extensivo con rotaciones, según criterios del productor se prefieren especies de pasto mejorado como; Toledo (Brachiaria brizantha cv. Xaraés) Marandú (Brachiaria brizantha Hochst. ex A. Rich) Mombaza (Panicum maximum c.v), acompañados con especies de pastos naturales como; Ratana (lschaemum Cilia) y gramas naturales, estas últimas por su gran resistencia al pisoteo y sobre pastoreo, el cual es uno de los principales problemas que presentan los pastizales.
Entre las actividades fitosanitarias que ellos realizan, está la vacunación para prevenir las enfermedades más comunes de la zona tales como: Pierna negra y Antrax. La vacunación se realiza dos veces, siendo con la entrada y salida del invierno (mayo y noviembre), además aplican una gama de desparasitantes como Ivomec, Ibermectina, Dectomac con dosis de $1 \mathrm{ml}$ por cada $50 \mathrm{~kg}$ de peso vivo y entre las vitaminas están $\mathrm{AD}_{3} \mathrm{E}$, complejo $\mathrm{B}$, calcio, entre otras. También aplican antibióticos como Oximec, Emicina, para tratar problemas de infecciones en el hato principalmente enfermedades como mastitis (inflamación de las glándulas mamarias).

La crianza de cerdos es de libre abierto, deambulan por todo el patio y los alrededores de las áreas agrícolas. En muchas ocasiones causan daños a los cultivos y esto provoca inconformidad entre los vecinos. Generalmente se crían cerdos de raza mejorada. La cantidad de cerdos por familia está ligada a la disponibilidad de alimento que posee cada familia, basada en maíz, yuca, desperdicios de cocina y suero de leche.

Para la sanidad de estos, aplican productos veterinarios principalmente desparasitantes y vitaminas, lo aplican cuando visualizan ciertos síntomas en los cerdos como: pelo poco brillante y erizo, pérdida del apetito y retraso del crecimiento. Según los comunitarios estos conocimientos los han adquiridos en capacitaciones y cuando hacen la consulta en las farmacias veterinarias. Los desparasitantes utilizados son el Ivomec, Ibermectina con dosis de $1 \mathrm{~mm}$ por cada $33 \mathrm{~kg}$ de peso vivo, recomendación que viene adjunta en los medicamentos.

Otras de las actividades importantes es la crianza de gallinas. El 100 por ciento de las familias dispone de gallinas criadas bajo el mismo sistema (libre abierto) alrededor de la casa. Generalmente son criollas y de acuerdo a la información de las mujeres, las prefieren porque crecen rápido y son resistentes a enfermedades. Su manejo es simple y tradicional, es decir, las gallinas deambulan libres por todos los espacios abiertos del patio, sin gallineros y duermen en las ramas de los árboles, minimizando costos por manejo. Con este sistema de crianza, las gallinas suplementan sus necesidades alimenticias consumiendo insectos y hierbas, sin embargo, hay algunas desventajas, como la pérdida de ellas a causa de depredadores como zorros, culebras, gavilanes y cerdos que las cazan. 
No se vacunan, ni desparasitan y como tratamiento contra enfermedades específicas, se les aplica medicamentos caseros, suministrándoles pastillas de sulfatiozol con jugo de limón real contra la gripe. Estas condiciones generan altos índices de mortalidad, principalmente en periodos de lluvia cuando las enfermedades respiratorias se agudizan y proliferan con mayor facilidad. La construcción de gallineros no es una práctica común dentro de estos sistemas de crianzas, Las aves se comercializan en pie. Son vendidas en las mismas comunidades o en los mercados municipales. Además de mejorar la dieta con huevos y carne que permiten variar la dieta, su comercialización permite a las familias adquirir productos industrializados como café, sal, jabón, azúcar, desodorantes, o bien ropa y calzado.

\section{Valoración económica y ambiental}

Para la valoración económica de cada una de las actividades productivas desarrolladas en los sistemas de producción se llevó a cabo mediante la aplicación de la metodología beneficio-costo que se explica como el retorno en dinero que se obtiene de cada unidad monetaria invertida. La falta de registros técnicos productivo por parte de los comunitarios limita conocer y llevar un control de los costos de cada una de las actividades realizadas durante el ciclo productivo, sin embargo, con el acompañamiento de los comunitarios, se hizo un ejercicio académico para el cálculo de los costos de cada actividad y determinar la rentabilidad de sus actividades productiva. Es importante considerar que estos sistemas funcionan bajo la lógica productiva de "empresa familiar", y su objetivo es generar ingresos económicos para la familia por medio de la comercialización del ganado mayor-menor y granos básicos.

En cuanto a la rentabilidad del subsistema agrícola, se determinó que esta actividad resulta factible, en el cultivo de maíz obtienen $C \$ 0,14$ córdobas por cada córdoba invertido, en cambio en el cultivo de frijol obtienen mejor rentabilidad con $C \$ 0,85$ córdobas por cada córdoba. Para el cultivo de yuca la rentabilidad es de $C \$ 1,01$ por cada córdoba invertido, según los cálculos este subsistema implementado por las familias es el más viable económicamente.

Además de las actividades agrícolas, las familias se dedican a la crianza de animales domésticos con fines productivos. La rentabilidad de la producción de leche, se calculó en base a los gastos que in- curren las familias en la manutención de una vaca lactante por un año, con una producción de 4 litros de leche diarios por 7 meses de lactancia como promedio. El precio promedio en el mercado es de $C \$$ 6,00 córdobas por litro, y obtienen una rentabilidad de $C \$ 0.5$ córdobas por cada córdoba invertido. Por otro lado, la mayoría de los productores, además de la producción de leche se dedican a la crianza de toretes de engorde. Según los cálculos obtienen $C \$ 1.41$ (córdobas) por cada córdoba invertido, se calculó en los costos de producción que implica la crianza (de un torete por 30 meses) periodo estimado que alcanza los $450 \mathrm{~kg}$ de peso vivo.

En relación con la valoración ambiental de los sistemas productivos, se pudo observar que hay serios daños ambientales a consecuencia de la deforestación, la conversión de áreas boscosas para la expansión de la ganadería y agricultura. La expansión de la frontera agrícola, la tala indiscriminada e ilegal de especies maderables han contribuido a la degradación ambiental, por lo que ha sido necesario la intervención de las autoridades ambientalistas como MARENA, INAFOR, Alcaldía, buscando la reducción del despale y promover la regeneración natural del bosque en las partes más altas donde nacen las quebradas, fuentes de agua potable, que abastecen a la comunidad.

Los problemas ambientales son muy notables donde la pérdida de la fertilidad natural del suelo es una de las problemáticas que afrontan las familias mestizas, obligando a los productores a la aplicación de altas dosis de fertilización química cada vez mayor. A su vez acelera el deterioro de la vegetación y la fauna, al bajar la producción natural de nutrientes. Es importante destacar que se visualizan huellas de que los ríos han disminuido su caudal y se han contaminado, al grado de convertirse en indicativo de la degradación ambiental de los sistemas productivos. Por otro lado, el uso excesivo de agroquímicos en las actividades agropecuarias crea resistencia a diferentes plagas que afectan los cultivos y a su vez producen mayor contaminación.

El bosque, a pesar de los grandes beneficios que ofrece, no es visto como un subsistema que les genera bienes y servicios o como un recurso que es necesario conservar, sino, es percibido como una oportunidad de generar recursos económicos mediante su explotación y conversión a áreas de cultivos o ganaderas. 


\section{Participación de la mujer en los sistemas productivos}

La familia en su conjunto hace posible el funcionamiento del sistema productivo, la participación familiar en las distintas tareas que demanda trabajar la tierra o crianza de los animales domésticos resulta ser de vital importancia. Se conoce mucho sobre el trabajo que realizan los hombres en las unidades productivas, y la responsabilidad de proveer los alimentos a su familia, sin embargo, no se habla mucho sobre el papel importante que las mujeres realizan para lograr estos objetivos o metas que en muchas ocasiones son atribuidos a los hombres. Según (FUNICA, 2009) esto se debe a la institucionalización del machismo con la cual se convive donde no se ha permitido visualizar el trabajo que realiza cada uno de los integrantes de una familia.

Por otro lado, el trabajo de campo, el cuido de los animales mayores como bovinos y equinos es responsabilidad propia de los hombres, esta idea aún prevalece, sin embargo, se pudo notar la participación de la mujer aportando en gran medida al funcionamiento de los sistemas productivos. Según el horario de trabajo y actividades en el hogar, la mujer suele ser el miembro de la familia que más trabaja en un día, llegando a laborar más de 14 horas diarias. Ella se ocupa del cuido del hogar, así como de una gran variedad de actividades productivas.

Es importante señalar, que las mujeres no ayudan a sus maridos, sino que trabajan igual y en ocasiones más que los hombres. Ellas participan en las labores de campo, que implica procesos de siembra, controles de malezas, cosechas y pos-cosecha. A pesar del gran aporte de la mujer con su trabajo existen diferencias significativas en cuanto a relaciones de poder y toma de decisiones (Tabla, 1), ya que generalmente el hombre es el que toma las decisiones sobre el uso, manejo y comercialización de los recursos.

Tabla No. 1. Acceso, control y toma de decisiones sobre los recursos productivos.

\begin{tabular}{lccc}
\hline \multicolumn{1}{c}{ RECURSOS } & PROP/CONTROL & USO/TRABAJO & DECIDE \\
\hline Acceso a Tierra & H & H.M & H \\
Ganado & H & H.M & H \\
Animales Menores & M & M & M \\
Herramientas & H & H. M & H \\
Crédito & H & H & H \\
Producción & H & H. M & H \\
Patio & M & H.M & M \\
\hline
\end{tabular}

Esto implica que las unidades productivas están siendo controladas por los hombres. FUNICA (2009) confirma este planteamiento al reflejar que solamente el $15,4 \%$ de la propiedad debidamente registrada está a nombre de mujeres y 1,7\% a nombre de la pareja y que el $94,3 \%$ de las mujeres propietarias tiene menos de 20 manzanas de tierra y el grupo con menor acceso a crédito en el país es el de las mujeres rurales de la Costa Caribe, con apenas el $18 \%$ de los préstamos otorgados, lo que significa que el hombre es el que tiene el control de los recursos, a como lo plantea la (FAO, 2005), impidiendo de esta manera a la mayoría de las mujeres poder decidir en las unidades productivas.

\section{Conclusiones}

El subsistema agrícola se caracteriza por ser semi-tecnificado con el uso común de una gran diversidad de agroquímicos y su producción está enfocada al autoconsumo y a la comercialización, así también el subsistema pecuario se define por ser extensivo y semi-tecnificado, siendo el ganado bovino la especie más relevante por la demanda de sus sub productos en el mercado.

En cuanto a la participación de la mujer, es amplia y orientada a las tareas del hogar, pero también a las actividades de siembra, manejo y cosecha- pos cosecha de los diferentes rubros, así como la crianza de los animales domésticos. Es importante señalar, que las mujeres no ayudan a sus maridos, sino que trabajan igual y en ocasiones más que los hombres.

Aunque, el sistema productivo mestizo es dinámico, activo, diverso y participativo, sin embargo, conlleva a ciertas repercusiones ambientales, considerando en uso de agroquímicos. Los problemas ambientales son muy notables donde la pérdida de la fertilidad natural del suelo es una de las problemáticas que afrontan las familias mestizas, obligando a los productores a la aplicación de altas dosis de fertilización química cada vez mayor.

Por consiguiente, es necesario implementar estrategias agroecológicas para la revitalización de los suelos de la Costa Caribe Norte de Nicaragua, así como prácticas productivas en equilibrio y armonía con la Madre Tierra para contribuir a la economía familiar comunitaria. 


\section{Bibliografía}

FUNICA (2009). Caracterización socioeconómica de la Región Autónoma del Atlántico Norte (RAAN) de Nicaragua. Fundación para el Desarrollo Tecnológico Agropecuario y Forestal de Nicaragua.

Frédéric M. (2010). La economía campesina colombiana de 1990. Cuadernos de Tierra y Justicia 2. $32 \mathrm{p}$.

FAO (2005). Género y sistemas de producción campesinos, lecciones de Nicaragua.
HART, R. (1980). Ecosistemas naturales del trópico húmedo San Andrés Colombia.

Jaime T. (1999). Análisis económico y financiero a nivel de finca. San Andrés, Colombia.

NITLAPAN (2010). Estrategias de adaptación al medio del Huracán Félix. Los casos de Butku y Awastingni. Región Autónoma de la Costa Caribe Norte. Nicaragua.

Ospina, A. (2008) Aproximación a la caracterización agroforestal: sintesis de una propuesta metodológica. 